\title{
UV1 Telomerase Peptide Vaccine
}

National Cancer Institute

\section{Source}

National Cancer Institute. UV1 Telomerase Peptide Vaccine. NCI Thesaurus. Code C104747.

A synthetic, peptide cancer vaccine directed against the human telomerase reverse transcriptase catalytic subunit (hTERT) with potential immunomodulating activity. Vaccination with the UV1 telomerase peptide may stimulate cytotoxic T-cells to recognize and kill telomerase-expressing cells. Telomerase, a reverse transcriptase normally repressed in healthy cells, is overexpressed in most cancer cells and plays a key role in cellular proliferation. 